East African Medical Journal Vol. 86 No. 6 June 2009

THERAPEUTIC MISCONCEPTION AND CLINICAL TRIALS IN SUB-SAHARAN AFRICA: A REVIEW

V.M. Lema, MBChB (Mak), MMed O/G (Nbi), Regional Medical Director, Family Health International, Africa Regional Office, Valley Road, P.O. Box 38835-00623, Nairobi, Kenya

\title{
THERAPEUTIC MISCONCEPTION AND CLINICAL TRIALS IN SUB-SAHARAN AFRICA: A REVIEW
}

\author{
V. M. LEMA
}

\begin{abstract}
Objectives: To identify possible existence of therapeutic misconception and its effects on clinical trials in sub-Saharan Africa.

Data source: Original research findings and reviews published in the English literature and author's professional experience with clinical trials in some East, Central and West African countries.

Design: Review of peer-reviewed articles.

Data extraction: Online searches and requests for reprints from corresponding authors and institutional subscription.

Data Synthesis: Information categorised accordingly.

Results: Therapeutic misconception, defined as a conflation by research subjects of research goals and those of routine health care is considered widely prevalent globally. The subjects misunderstand the disclosures during consenting process and enroll hoping to derive personal benefits from the study. Though no study has looked at therapeutic misconception specifically in sub-Saharan Africa, available evidence suggests that it is prevalent. Therapeutic misconception is incompatible with informed voluntary consent. It may affect participation in clinical trials, subjects' safety and well-being and possibly the research findings.

Conclusions: There is need for studies to identify the prevalence and effects of therapeutic misconception in the region. Researchers in sub-Saharan Africa should be aware of its existence, thus design trials in which it will not have significant effects and strengthen the consent process to reduce it.
\end{abstract}

\section{INTRODUCTION}

Clinical research is essential for development of new knowledge related to health promotion, prevention and clinical treatment of health conditions and the understanding of various health problems. Over the past 15 to 20 years there has been an upsurge in the number, scope and size of clinical trials which has been accompanied by globalisation thereof (1). The spatial geographical distribution of disease conditions to be studied, the need to test the safety and efficacy of new therapies in diverse situations and populations due to potential effects of social, ecological and genetic disparities have influenced internationalisation of clinical research (2-4). Other factors include the need to reduce trial costs by increasing subject recruitment rates and shortening trial periods.

International ethics on new therapies or devices require that before they can be routinely used in clinical situations or for prevention of diseases they must be rigorously tested in human beings with or at risk of acquiring the respective health condition under well controlled situations. Randomised clinical trials (RCTs) are considered "the gold standard" in clinical research as they provide the most reliable data for evidence-based clinical / health care. Randomisation of the subjects among the various treatment arms of the study reduces the effects of factors other than the intervention/therapy being tested thus promoting confidence in the results thereof. In these trials the investigational drug, vaccine, device or therapeutic approach/ preventive strategy is compared to either a standard one or a placebo (5).

For preventive trials such as vaccine or HIV prevention strategies such as male circumcision, healthy volunteers who are individuals at risk of acquiring the respective conditions are invited to participate in the trials through local media outlets, messages from health care providers or health facility notice boards. In cases of therapeutic trials such as those on ARVT, antimalarials, antibiotics, cancer chemotherapy, or surgical techniques various categories of patients with the respective health condition are likewise invited to participate, by their attending physicians or researchers. In either case the potential subjects must give informed consent voluntarily before they can be enrolled into the trials (4-7). This must be achieved through an 
interactive, continuous process between a designated member of the research team and the patient/ subject before, during and even beyond the trial period depending on the trial (7). The goal is to ensure that the trial participants achieve informed decisions and implement them appropriately. Faden and Beauchamp gave three stages of the consent process;

(i) Transmission and reception of relevant information

(ii) Comprehension of the transmitted information

(iii) Use of the information to arrive at a decision.

The disclosure should include among other issues:

(i) The nature and purpose of the study.

(ii) The procedures involved including randomisation, use of placebos and why; the laboratory tests, follow up (home) visits - how long and why?

(iii) Any foreseeable risks and benefits in participating.

(iv) Appropriate and available alternatives to participation including non-treatment in some cases.

(v) That participation is voluntary and one may withdraw at any stage of the study without prejudice.

(vi) Explanationon the differencesbetween research (clinical trial) and routine clinical/health care, especially the fact that one may not receive an effective therapy and the loss of personalised care inherent in clinical care $(6,7,9,10)$.

The information must be disclosed in a manner and language that will facilitate understanding by the potential subjects. The subject must be competent to make decision based on their comprehension of the information and voluntarily decision to either participate in the trial or not.

There have been concerns though whether and how much of the disclosures research subjects understand and retain or whether they really appreciate the implications of their decision to participate (11-13). Either as a result of the nature of disclosures or subjects' comprehension thereoforboth, some patients / subjects may not quite understand the differences between research and routine health/ clinical care and therefore the implications of their enrollment. The Belmont Report (6) defines research by its purpose that is "an activity designed to test an hypothesis, permit conclusions to be drawn and thereby contribute to generalisable knowledge". When research subjects conflate between the goals of research / clinical trials and those of clinical / health care they are said to have "therapeutic misconception" $(9,14-17)$.
This article is a review of evidence on the prevalence and effects of therapeutic misconception in the English literature. It also explores the possible existence of the phenomenon and its potential effects on clinical trials in sub-Saharan Africa, as well as makes recommendations on strategies to address the gap in information from the region and reduce it.

\section{The therapeutic misconception}

Definition: Therapeutic misconception was first described by Appelbaum et al (14) as the "mistaken belief by research subjects that research like therapy they have received before, is designed and will be executed in a manner of direct benefit to them". Lidz et al (16) refined it thus "therapeutic misconception occurs when a research subject fails to appreciate the distinction between the imperatives of clinical research and of ordinary (routine) treatment and therefore inaccurately attributes therapeutic intent to research procedures".

The National Bioethics Advisory Commission (NBAC) defined therapeutic misconception as "the belief that the purpose of a clinical trial is to benefit the individual patient, rather than to gather data for the purpose of contributing to scientific knowledge (18). Macklin (19) characterised therapeutic misconception as 'the belief that research is promising treatment intended to benefit study participants". Lately Henderson et al (20) offered a new definition that "therapeutic misconception exists when individuals do not understand that the defining purpose of clinical research is to produce generalisable knowledge, regardless of whether the subjects enrolled in the trial may potentially benefit from the intervention under study or from other aspects of the clinical trial.

There are some disagreements though as to the definition of therapeutic misconception (20, 21). While acknowledging that some consider over estimation of clinical benefits from an experimental intervention as well as under estimation of potential risks of harm to be part of therapeutic misconception. Horng and Grady (21) describe different scenarios of misunderstanding by research subjects. They drew distinctions among "therapeutic misconception", "therapeutic mis-estimation" and "therapeutic optimism". They noted that therapeutic misconception (TM) has been used rather loosely to refer to any number of misunderstandings that subjects may have in the research context, but agree with others $(14,17,22,23)$ that therapeutic misconception is a conflation by research subjects of research goals with those of clinical care. They however define "therapeutic mis-estimation" as "a misunderstanding of the probability of benefits and / or harm in research", while "therapeutic optimism" is "hope for the best outcome". They contend that misunderstanding the nature and intent of research is 
most ethically problematic, while mis-estimating the probability of direct benefits may be less worrisome (21).

Simon et al (24) described a similar scenario in preventive clinical trials noting the similarities and differences between them and therapeutic trials. They referred to it as "preventive misconception" (PM) and defined it as "the over estimation of probability or level of personal protection that is afforded by being enrolled in a trial of a preventive intervention".

Origins: Clinical trials share certain characteristics with routine medical / health care which may blur the boundary between research and therapy or preventive health care services to not only the patients / subjects but also the health care providers/investigators.

Most clinical trials are often carried out within local health facilities or established research facilities. The same health facilities are used by the local community from which the patients/subjects will be drawn for routine health care including health education and preventive services. The research investigators are drawn from amongst the health care providers in the same facilities. Clinical trials use drugs or treatment devices, the same as in routine health care. The procedures used in clinical trials such as history taking, physical examination, laboratory tests are also used in routine medical care. The subjects for phase III therapeutic clinical trials are patients with the same health condition under study and who will have come to the same facilities seeking medical attention for their ailments. For preventive clinical trials the subjects are healthy individuals at risk of acquiring the health condition under study. Both groups may be just too eager to get a cure for their condition or prevention of a dreaded and life-threatening condition. In either case the subjects expect that physicians/health care providers will only invite them to participate in a clinical trial when such participation would be in their best interest while others may enroll with the belief that the drugs/intervention offered in clinical trials are of a superior quality $(15,25)$.

Clinical trials are a fairly new phenomenon in sub- Sahara Africa (SSA). Many people especially in rural areas may not have had previous experience with clinical trials/ research, whereas mostmay have sought medical care either for themselves or their relations in the same facilities. Health facilities such as hospitals, health centres are generally known for treatment and / or preventive care, rather than for clinical trials / research. Health care providers including doctors, nurses, laboratory technologists are by their training expected to provide the best care available to their patients and patients trust their professionalism.

Prevalence: Studies conducted in the more developed countrieshaveshown that therapeutic misconception is widely prevalent in clinical trials $(9,19,25,26)$. Lidz et al (9) stated that therapeutic misconception is a widely recognised problem in informed consent, but did not specify the prevalence thereof. In their later study Lidz et al (16) in which they interviewed 225 subjects enrolled in 44 clinical protocols $62 \%$ of them were judged to manifest therapeutic misconception. Appelbaum et al (15) reported that $69 \%$ of patients in their survey were unaware of the fact that their assignment to treatment interventions would be randomised and $40 \%$ expected their assignment would be made on the basis of their therapeutic needs.

Personal benefits have been reported to be the major motive behind research participation even in studies with little or no prospect of benefit $(25,26)$. Riecken and Ravich (13) reported that 75\% of their subjects' reason for participating in a clinical trial was desire for personal benefit. Penman et al (27) interviewed 144 cancer patients in a number of phase II and III clinical trials, and observed that although the consent documents clearly indicated that benefits could not be assured, $43 \%$ of the subjects stated that they had no doubts at all about personal benefits from the study interventions. Schaeffer et al (26) reported that $100 \%$ of subjects in phase I studies described their studies as having both research and treatment aims. Ellis et al (28) observed that $74 \%$ of subjects in their survey thought the doctor would ensure that they receive the best treatment available in a randomised clinical trial.

Although there has been no study specifically looking at or which has identified prevalence of therapeutic misconception in (SSA), there is lot of evidence indicating poor understanding of research goals and / or informed consent disclosures. Joubert et al (29) in their study on consent for a vitamin A trial in South Africa observed that there was generally poor knowledge about the trial and $92.3 \%$ of the subjects felt they had to stay in the study to get good medical care. In a PMTCT project in Coted'Ivoire, Ekoueviet al (30) found that only $48 \%$ of the participants reported to have understood the information disclosed. Krosin et al (31) in their study on consent process in Mali noted that $93 \%$ of the subjects did not understand they were enrolled in an investigation as opposed to receiving therapy, implying that they hoped to receive treatment for their conditions by participating in the trial. More recently Oduroet al (32) in Ghana, reported that $70 \%$ of the study participants gave personal direct benefits as their reason for participating in the clinical trial and only $20 \%$ recalled being told about study-related risks.

Factors influencing therapeutic misconception: It is agreed that therapeutic misconception exists when a research subject fails to appreciate the distinction between the imperatives of research and those of 
routine clinical/ health care and therefore inaccurately attributes therapeutic intent to research procedures. However subjects may understand the goals and methods of research project but still claim therapeutic motivation for participating, while others may fail to understand the aims of a particular research project or the procedures therein, yet they do not attribute therapeuticintent for their enrollment. Certain factors have been shown to influence the occurrence of therapeutic misconception among patients / subjects participating in clinical trials.

The title of the protocols as well as terms used to describe the trial: Sometimes brand names or acronyms are used in the title of the protocol such as ALIVE, PROVED, MAGIC. These may suggest the outcome of the trial before it starts. Terms like "therapeutic trial" "treatment trial" "HIV prevention trials", or titles which do not clearly spell out what the trial entails may mislead the potential participants. Other terms commonly used in clinical trial protocols such as "randomisation" and "placebo" have been shown not to be well understood or interpreted by the lay population from which majority if not all of clinical trial subjects are drawn. Featherstone and Donovan (33) reported that subjects who had participated in a clinical trial interpreted "at random" to mean "without purpose", while Waggoner et al (34) in their study involving a general public sample found that only $22 \%$ of them knew the meaning of the word "randomly". In a study on decision-makers, that is, parents and guardians for childhood leukaemia trials, Kodish et al (35) reported that $50 \%$ of them did not understand the concept of randomisation.

The consent documents: The language used in consent documents may be above the literacy level of most potential subjects. They may therefore not being comprehensibletothem(36). Evenwhen the documents are translated into local languages as required by ethics committees (ECs) they may still not be understood by majority of the potential subjects, or the meaning therein may be lost. At the same time some terms such as "random" "placebo" do not haveequivalent in some languages. The contents of the consent documents are very critical in facilitating decision making process for the potential subjects $(8,13,36)$. Some documents donot state the purpose of the study or what will be done, the benefits and/ or risk of harm to participants. Horng and Grady (21) in a study of 272 consent forms for phase I therapy studies found that $99 \%$ of them explicitly stated that the purpose was experimental research and 92\% indicated that the primary aim was to assess safety. On the other hand, Henderson et al (37) in a survey of 39 informed consent documents for phase I gene transfer trials, observed that $74 \%$ did not clearly convey to the subjects that benefit was neither likely nor expected, but instead used the term "indeterminate".
The way the messages therein are packaged and or disseminated may also be a contributory factor. It has been shown that many patients/subjects have deficiency in correctly interpreting risk and/or benefits expressed as percentages (37). When male circumcision for prevention of HIV infection was being rolled out in some countries in SSA for example, some individuals interpreted the $60 \%$ protection to mean that if they reduced their sexual partners by a factor of $40 \%$ they would be okay! Some consented with that belief in mind. There may at times be more emphasis or over-estimation of the benefits of participation, with exclusion or downplay of potential risks, adverse events of the intervention or procedures involved in the trial.

The subjects: In phase III therapeutic clinical trials, the subjects are patients with varying degrees of the clinical condition under study. Some may have had previous treatment for the same condition with varying success rates and perceptions thereto. Others may be seeking treatment for their conditions for the first time. The psychological reaction/response to the illness varies a lot among the patients and/or their close family relations or friends. For phase III preventive clinical trials the subjects are healthy volunteers at risk of acquiring the health condition under study. Their knowledge of the disease or a friend or relation who has suffered from it as well as their fears and perceived risk of acquiring the disease may influence their decision to volunteer. Some may enroll out of fear of the health condition, its nature and desperation. Many subjects cite the hope of benefit even when there is little hope thereof, as a prominent reason foe enrolling in clinical trials (32). Patients / subjects may enroll in a clinical trial with the belief that they will receive better treatment/ care (12), that their assignment to a treatment arm or the intervention will be tailored to their best interests even when told explicitly that randomisation will be used $(9,25)$.

Understanding of disclosure and reaction thereto varies a lot among individuals. Aaronson et al (38) observed that overall understanding of disclosures correlated with age and education, being better among the young and more educated. Lidz (23) reported that $62-86 \%$ of subjects manifested substantial error in appreciating the issues in participating in clinical trials. Others may not understand the scientific methodology (15). At times the patient/ subject may misinterpret the disclosure.

Having cognitive ability to make rational decision and receiving adequate information does not necessarily lead to an adequate understanding of issues athand. Most individuals make context-bound rather than context-free decisions. Their illness and severity thereof may influence their decision-making process, as shown by Schaeffer et al (26). Patients / 
subjects' trust (misplaced trust) in the health care providers/researchers and institutions involved either because of previous experience or standing in society may also influence therapeutic misconception. It has been shown that some categories of people may have low or high tendency to fall prey to therapeutic misconception. African-Americans are said to have low tendency as they are more suspicious of clinical researchers and have less favourable attitudes towards research $(25,39)$. This may also apply to some populations based on their experience with clinical trials or health care providers/institutions.

The investigators and institutions: The subjects are recruited from among the general population or patients by their health care providers or invited/ encouraged through institutional bulletins or adverts. The investigators themselves may confuse research and therapy in their informed consent process with potential subjects. Professional identify, that is, as physicians, may interfere with their decision to recruit their own patients into clinical trials due to a conflict between their allegiance to patient care and clinical research/trial. Patients may thus enroll in a study on invitation or suggestion by their physicians trusting that they will put their health care interest before anything else.

What the researchers disclose to potential subjects is central to their decision-making. Some may deliberately be selective in what they disclose. A study by Verheggen et al (40) showed that investigators emphasised aspects of the trial they expected patients/subjects would understand easily and not those they would have difficulty in understanding such as study design, randomisation and selection procedures. Sometimes the researchers rarely plan meaningful discussion beyond the study purpose and procedures or what is contained in the standard consent documents. Efforts are not made to explain the nature of the research, differences between the goals thereof and those of routine clinical/health care as well as implications of their participation. Lidz (23) state that researchers are often unwilling or even fail to acknowledge to themselves that the care they offer in a clinical trial is not optimised for the patient/ subjects' benefit which may blur the consent process. Henderson et al (37) stated that the ways in which the investigators describe the likelihood of benefits in a study have a strong influence on occurrence of therapeutic misconception in their study subjects. Some may use or explain probability of risk or benefits in percentages or proportions in a manner that distort the reality.

At times researchers fail to disclose or discuss aspects of the research which limit subjects personalised care in clinical trials (13), or may use the consent process in a way that favours subjects accrual in clinical trials so as to realise the target numbers in the shortest possible time.

The fact that clinical trials are conducted in established health facilities where subjects mix with other patients and the fact that these institutions may have a good reputation may contribute to misplaced trust that they will offer state of the art care.

Effects of therapeutic misconception: As stated above therapeutic misconception exists when a research subject inaccurately attributes therapeutic intent to research participation. The subjects confuse the goals of research with those of routine health care. Their enrollment into the study is therefore premised on the belief that they will derive personal benefits therefrom. Therapeutic misconception may therefore affect the informed consent given for enrollment as well as the subjects' participation in the study, thus presenting an ethical problem in clinical trials (9).

Effect on informed consent: Truly informed voluntary consent implies that the research participants have understood the essence of the study, their rights and responsibilities as research participants. For it to achieve its goals of promoting autonomy and decision-making, it is critical that patients/subjects not only understand the elements of the disclosures but also appreciate its applicability to their individual situations or that of their children in case of parents or guardians consenting on behalf of minors. When people do not understand or accept scientific explanation of health and disease and by extension clinical trials obtaining informed consent can be a daunting challenge (18).

While some subjects may clearly understand the disclosure during consenting process and give their consent voluntarily, they may still have therapeutic misconception if their goal for participating is personal benefit. Therapeutic/ preventive misconception represents lack of meaningful informed consent and participants may not appreciate the consequences of their decisions (41). Subjects manifesting therapeutic misconception can therefore not be said to have given informed and voluntary consent for their participation.

Effect on participation: It is agreed that truly informed and voluntary consent is a pre-condition for ethical involvement of human subjects in clinical trials $(4,6$, 16). Informed consent is among other things meant to ensure that only eligible individuals according to the nature and goals of the research protocol are recruited and enrollment into the respective study. Therapeutic misconception may therefore affect participation in a clinical trial in a number of ways. Subjects who may not be eligible as per protocol requirements may be inadvertently enrolled. It has 
been suggested therapeutic misconception may lead to an increase in recruitment (25). However no study has specifically evaluated it.

Subjects may be exposed to harmful effects of the study procedures or the intervention and/or sustain adverse effects which they may neither relate to their participation in the trial nor report back to the research team as required. Desperate patients/ subjects may be induced to accept unreasonable risks on the false assumption that their situation "can not get any worse", thus casting a shadow over demands for beneficence and respect (17).

When the expected benefits either a cure or prevention of the health condition is not realised, the participants / subjects may lose trust in the health facility / research institution as well as their health care providers / researchers. They may feel let down by their health care providers. This may not only affect future research/clinical trials but also their health seeking behaviours $(25,39)$. When the subjects discover that they are not on the interventions they had hoped to be put on, they may on top of losing trust in the team, drop out from the study, thus potentially affecting the study results (39).

For preventive trials subjects who have preventive misconception may exhibit behavioural changes which may put themselves as well as other people at greater risk of acquiring the disease (24, $41,42)$. This is particularly worrying with regards to HIV prevention trials interventions.

\section{DISCUSSION}

Internationalisation of clinical trials, while a relatively new phenomenon is bound to increase in number, scope and size for among other reasons economic concerns related thereto (1), emergence of new disease conditions and need to test efficacy and safety of new drugs, therapeutic devices and preventive strategies in diverse populations and conditions $(2,3)$. Some disease conditions such as HIV / AIDS, tuberculosis, malaria, childhood diarrhoeal diseases, while global they are more prevalent and with more serious sequelae in developing countries, SSA included. Hence the need to conduct relevant clinical trials in the region.

Despite international guidelines which govern research involving human beings, there have and continue to be considerable controversies around ethics of clinical trials originated, sponsored or conducted by industrialised countries and carried out in developing countries $(43,44)$. The controversies have been brought to the fore by clinical trials on HIV in developing countries. It is however not limited thereto.

Although therapeutic misconception is widely prevalent among research subjects globally $(9,16$,
$26,27)$, there have been no studies specifically on this phenomenon in SSA. However several studies conducted in the region over the past ten or so years, have documented poor understanding of various aspects of research protocols including the consent disclosures. Ekouevi et al (30) reported that only $48 \%$ of subjects understood the information disclosed. Krosin et al (31) in Mali found that $93 \%$ of subjects thought they were receiving routine therapy, implying that they did not understand what they were told during the consent process regarding the nature of the trial. Moodley et al (45) reported that only 21\% participants of their study in South Africa could recall being randomly allocated to treatment arms of the study and $19 \%$ of those on a placebo-controlled study knew what placebo meant. Only $10 \%$ of participants in a vaccine trial in the Gambia understood that a placebo would be used (46), while Oduro et al (32) observed that only $20 \%$ of the subjects in a trial in Ghana recalled being told about study-related risks during consent process.

Therapeutic misconception exists when research subjects conflate the imperatives of research with those of routine health care, thus attributing therapeutic intent to research procedures $(9,15-17)$. The question is which and to what extent does the misunderstanding observed amongst study participantsinSSAconstitute therapeutic misconception < If the phenomenon is widely prevalent in the developed countries, with their higher literacy levels, better health systems and facilities, it may not be far-fetched to believe that it is equally or even more prevalent in SSA. Krosin et al (31) averred that the degree of miscomprehension observed in their study in Mali was more than that found in similar studies in developed countries.

This may not be surprising as the factors known to influence occurrence of therapeutic misconception, such as titles and terms used to describe the research protocols, the language used and contents of consent documents, the nature and severity of the health condition under study and subjects desperation for cure/prevention, or their trust in their health care providers/investigators and/or health/research institutions, may be of particular concern in SSA, with its unique situations such as poverty, poor / weak health systems and socio-cultural factors. Karim et al (12) in their study in South Africa found that $22 \%$ of the participants enrolled with the hope of receiving better medical care. Molyneux et al (47) described the role of trust by parents in misunderstanding of studies for which they consented for their children to participate in Kenya. Life-threatening conditions such as HIV / AIDS, malaria, cancer, which are prevalent in SSA against a background of non-availability of better alternative or any treatment options and the conditions under which clinical trials are carried out compared to those pertaining to routine health care 
may influence potential subjects' decision-making to enroll in clinical trials thus contributing to therapeutic misconception (48).

Of greater concern with regards to therapeutic misconception inSSAisits potential effect on informed consent and subjects' participation in clinical trials and the quality of research findings. Joubert et al (29) concluded that with the poor knowledge amongst participants in their study in South Africa, they could not have been considered to be informed. Patients/ subjects will consent to participate in trials with the hope and belief that they will derive personal benefits therefrom. They may therefore beexposed to researchrelated risks which they may neither appreciate nor report. In SSA with a myriad of disease spectrum, some with overlapping symptoms and varied cultural beliefs on causality of diseases and tendency to seek traditional medicine for treatment, this could pose a serious problem. An individual believing that he/she or his/her children have been protected against a particular disease, may wrongly assign symptoms of the disease to other conditions or blame them on witchcraft, evil spirits, etc and may not seek medical help. When they discover that they are not receiving the intervention they expected to get on enrolling, they may become bitter and angry, lose their trust in the investigators and institutions and may drop out from the study. If the proportion of those who drop out or those with serious adverse events do not report back to the research team is significant it could have an impact on the research findings. Others may not participate in future clinical trials or may change their health seeking behaviour henceforth because they feel let down by the people they trusted. For preventive clinical trials the perceived notion of protection, for example, against HIV infection, may lead to behavioural changes. The subjects may adopt risky behaviours as shown by Bartholow et al (21) and Chesney et al (42). These observations are of major concern as we scale up HIV prevention trials and strategies in SSA.

\section{CONCLUSIONS AND RECOMMENDATIONS}

The prevalenceand effects of therapeutic/ preventive misconception in SSA remains largely unknown despite the overwhelming evidence thereof from developed countries. This may be due to the fact that clinical trials are a fairly new phenomenon in the region, or lack of awareness of the phenomenon by researchers in the region.

Based on available evidence on the extent of misunderstanding of research related issues in SSA, some of which may contribute to therapeutic misconception, it is not far-fetched to infer that it is equally if not more prevalent and that the effects thereof may have serious impact on subjects' informed consent, research participation and quality of research findings.

Sadly despite overwhelming evidence on the prevalence of therapeutic misconception and numerous calls to design and implement strategies to either eliminate or reduce it, therapeutic misconception continues to be tolerated and even fostered in some cases.

Therapeutic misconception is incompatible with adequate informed consent and undermines the trustworthiness of clinical trials and research in general.

Cognisant of the foregoing, it is recommended that:-

(i) There is need for research studies in different settings and communities in SSA to identify the prevalence, determinants and effects of therapeutic misconception.

(ii) Researchers should design clinical trials in such a manner that therapeutic misconception will have limited consequences that is, avoid as much as possible the use of placebos in randomised clinical trials.

(iii) Researchers should ensure that they provide adequate disclosures during consenting process in particular explain the differences between research and routine clinical/health care and loss of personalised care in clinical trials.

(iv) Researchers should ensure the consenting process is continuous throughout the study period and not a one-off event before enrollment.

(v) There is need to create public awareness on the differences between research and routine health care, risks and benefits of participating in clinical trials, the subjects rights and responsibilities.

(vi) There is need to develop and build capacity of local Ethics Committees and Institutional Review Boards so that they may adequately review research protocols and consent documents, thus protecting subjects' rights, safety and well-being.

(vii) There is need to create awareness amongst researchersinSSAon the existence of therapeutic misconception, its impact on clinical trials and possible strategies to reduce it.

\section{REFERENCES}

1. Glickman, S.W., McHutchison, J.G., Peterson, E.D., et al. Ethical and scientific implications of the globalization of clinical research. N. Engl.J.Med.2009; 360: 816-823. 
2. Califf, R.M. Simple principles of clinical trials remain powerful. J. Amer. Med. Assoc. 2005; 293:489-491.

3. Larson, H.N., Zhou, J., Chen, Z., et al. Structural and functional consequences of coenzyme binding to the inactive Asian variant of mitochondrial aldehyde dehydrogenase: roles of residues 475 and 487. J. Biol. Chem. 2007; 282:12940-12950.

4. Council of the International Organisation of Medical Sciences. International Guidelines for Biomedical Research Involving Human Subjects. At http://www. cioms.ch/frame_guidelines_nov_2002.htm

5. WHO.Guidelines for GCP for trials on pharmaceutical products. WHO Technical ReportSeries no. 850, 1995. WHO Geneva.

6. TheBelmont Report. Ethical Principles and Guidelines for the Protection of Human Subjects of Research. Available at: http://ohrp.osophs.dhhs.gov/humansubjects Iguidance/belmont.htm.

7. The Nuremberg Code. Available at $h t t p: / / o h s r . o d . n i h$. gov/guidelines / nuremberg.html.

8. Faden, R.R. and Beauchamp, T.L. Ahistory and theory of informed consent. New York: Oxford University Press. 1986: 235-273.

9. Lidz, C.W. and Appelbaum, P.S. The therapeutic misconception: problems and solution. Med. Care. 2002; 40(9 Suppl):V55-63.

10. Fried,C. Medical experimentation: personal integrity and social policy. New York, NY; American Elservier Publishing Co. 1974,

11. Hill, Z., Tawiah-Agyemang, C., Odei-Danso, S. and Kirkwood, B. Informed consent in Ghana: what do participants really understand? J. Med. Ethics. 2008; 34: 48-53.

12. Karim, Q.A., Karim, S.S.A., Coovadia, H.M. and Susser, M. Informed consent in a health care setting: is it truly informed and truly voluntary? Am. J. Pub. Health. 1998; 88: 637-40.

13. Riecken, H.W. and Ravich, R. Informed consent for biomedical research in Veterans Administration Hospitals. J. Amer. Med. Assoc. 1982; 248: 344-348.

14. Appelbaum, P.S., Roth, L.H. and Lidz, C.W. The therapeutic misconception: Informed consent in psychiatric research. Int. J. Law Psych. 1982; 5: 319-329.

15. Appelbaum, P.S., Roth, L.H., Lidz, C.W., et al. False hopes and best data: consent to research and the therapeutic misconception. Hastings center Report 1987; 17: 20-24.

16. Lidz, C.W.,Appelbaum, P.S., Grisso, T. and Renaud, M. Therapeuticmisconception and the appreciation of risks in clinical trials. Soc. Sci. Med. 2004; 58: 1689-1697.

17. Fried, E. The therapeutic misconception, beneficence and respect. Account. Res. 2001; 8: 331- 348.

18. National Bioethics Advisory Commission (2001). Ethical and policy issues in international research: clinical trials in developing countries. Available at http:/ / bioethics.georgetownedu.nbac/pub-html.

19. Macklin, R. Understanding informed consent. Acta Oncologica. 1999; 88: 83-87

20. Henderson, G. E., Churchill, L. R., Davis, A. M., et al. Clinical trials and medical care: defining the therapeutic misconception. PLoS Med. 4: e324. doi:10.1371/journal pmed.0040324.

21. Horng, S. and Grady, C. Misunderstanding in clinical research: distinguishing therapeutic misconception, therapeutic mis-estimation and therapeuticoptimism. IRB 2003; 25: 11-16.

22. Appelbaum, P.S., Lidz, C.W. and Grisso, T. Therapeutic misconception in clinical research; frequency and risk factors. IRB. 2004; 26: 1-8.

23. Lidz, C.W. The therapeutic misconception and our models of competency and informed consent. Behav. Sci. Law. 2006; 24: 535-546.

24. Simon, A. F., Wu, A.W., Lavori, P. W. and Sugarman, J. Preventive misconception: Its nature, presence and ethical implications for research. Am. J. Prev. Med. 2007; 32: 370-374.

25. Advisory Committee on Human radiation Experiments (ACHRE). Final Report. Washington, DC: US Government Printing Office; 1995;

26. Schaeffer, M.H., Krantz, D.S., Wichman, A., et al. The impact of disease severity on the informed consent process in clinical research. Am. J. Med. 1996; 100: 261-268.

27. Penman, D.T., Holland, J.C., Bahna, G.F., et al. Informed consent for investigational chemotherapy: patients' and physicians' perceptions. J. Clin. Oncol. 1984; 2: 849-855.

28. Ellis, P.M., Dowsett, S.M., Butow, P.N., et al. Attitudes to randomized clinical trials amongst out-patients attending a medical oncology clinic. Health Expect. 1999; 2:33-43.

29. Joubet, G., Steinberg, H., der Ryst, E. and Chikobvu, P. Consent for participation in the Bloemfontein Vit. A trial: How informed and voluntary? Am. J. Pub. Health. 2003; 93: 582 - 584.

30. Ekouevi, D.K., Becquet, R., Viho, I., Bequet, L., et al Obtaining informed consent from HIV infected pregnant women, Abidjan, Cote d'Ivoire. AIDS. 2004; 18:1486-1488.

31. Krosin, M.T., Klitzman, R., Levin, B., et al. Problems in comprehension of informed consent in rural and peri-urban Mali, West Africa. Clin Trials. 2006; 3: 306-313.

32. Oduro, A.R., Aborigo, R.A., Amugsi, D., et al. Understanding and retention of the informed consent process among parents in rural Northern Ghana. BMC Medical Ethics. 2008; 9:12 doi:10.1186/1472-6939-9-12.

33. Featherstone, K. and Donovan, J.L. Random allocation or allocation at random? Patients' perspectives of participation in a randomised controlled trial. Brit. Med. J. 1998; 317(7167):1177-1180.

34. Waggoner WC; Mayo DM. Who understands? A survey of 25 words or phrases commonly used in proposed clinical research consent forms. IRB. 1995; 17:6-9.

35. Kodish, E., Eder, M., Noll, R.B., et al.Communication of randomization in childhood leukemia trials. J. Amer. Med. Assoc. 2004; 291:470-475.

36. Kimmelman, J. and Levenstadt, A.E. Elements of style: Consent form, language and the therapeutic misconception in phase 1 gene transfer trials. Hum. Gene. Ther. 2005; 16:502-508.

37. Henderson, G.E., Davis, A.M. and King, N.M. Vulnerability to influence: a two way street. Am. J. Bioeth. 2004; 4: 50-52.

38. Aaronson, N.K., Visser-Pol, E., Leenhouts, G.H. Telephone-based nursing intervention improves the 
effectiveness of the informed consent process in cancer clinical trials. J. Clin. Oncol. 1996; 14:984-996.

39. Harris, Y., Gorelick, P.B., Samuels, P. and Bempong, I. Why African-Americans may not be participating in clinical trials. J. Natl. Med. Assoc. 1996; 88: 630-634.

40. Verheggen, F.W.S.M., Jonkers, R. and Kok, G. Patients' perspectives on informed consent and the quality of information disclosed in clinical trials. Patient Educ. Couns. 1996; 29: 137-153.

41. Bartholow, B.N., Buchbinder, S., Celum, C., et al. VISION/VAX004 Study Team. HIV sexual risk behavior over 36 months of follow-up in the world's first HIV vaccine efficacy trial. J. Acquir. Immune Defic. Syndr. 2005; 39: 90-101.

42. Chesney, M.A., Chambers, D.B. and Kahn, J.O. Risk behavior for HIV infection in participants in preventive HIV vaccine trials: a cautionary note. J. Acquir. Immune Defic. Syndr. Hum. Retrovirol. 1997; 16: 266-271.

43. Annas, G.J. and Grodin, M.A. Human rights and maternal-foetal HIV transmission prevention trials in Africa. Am. J. Public Health. 1998; 88:560-563.
44. Angell, M. The ethics of clinical research in the third world. N. Engl. J. Med. 1997; 337: 847-849.

45. Moodley, K., Pather, M. and Myer, L. Informed consent and participant perceptions of influenza vaccine trials in South Africa. J. Med. Ethics. 2005; 31:727-732.

46. Leach, A. An evaluation of the informed consent procedure used during a trial of a Haemophilus influenzae type B conjugate vaccine undertaken in the Gambia, West Africa. Soc. Sci. Med. 1999; 48: 139148.

47. Molyneux, C.S., Peshu, N. and Marsh, K. Trust and informed consent; insights from community members on the Kenyan Coast. Soc. Sci. Med. 2005; 61: 14631473.

48 Lema, V.M., Mbondo, M. and Kamau, E.M. Informed consent for clinical trials: A review. East. Afr. Med. J. 2009; 86: 133 - 142 . 